\title{
Towards Domain-Independent Conflict Resolution Tools
}

\author{
Davide Carneiro \\ Universidade do Minho \\ dcarneiro@di.uminho.pt
}

\author{
Paulo Novais \\ Universidade do Minho \\ pjon@di.uminho.pt
}

\author{
José Neves \\ Universidade do Minho \\ jneves@di.uminho.pt
}

\begin{abstract}
Given the current state of the legal systems, Online Dispute Resolution tools are being regarded as an alternative way to solve conflicts out of courts, namely under virtual environments. However, the use of these tools is still relatively restricted as they are still few in number and very domain-cantered. Indeed, abstract and conceptual tools whose building blocks could be adapted for particular use would foster the development of ODR systems. In this paper we present this novel line of attack, in which an agent-based architecture is used with the support of an ontology to build an abstract and formal ODR system, independent of the legal domains, but specific enough to be applied to concrete ones. Functionality reuse is maximized, making architectures simpler to implement and to expand.
\end{abstract}

\section{Introduction}

The current habits and routines of our society have been shaped by the recent unprecedented technological evolution. One of the notorious examples is the rising percentage of contracts, in the most different fields, that are now signed under an electronic or virtual environment [1], of which web-sites like the ones of Amazon and e-Bay are the best examples. However disputes can also arise in an electronic setting, which poses some new challenges to the established conflict resolution mechanisms (traditionally litigation in court).

When solving disputes out of court, essentially the ones arising out of electronic contracting, Online Dispute Resolution (ODR) must be considered. ODR techniques allow already traditional alternative dispute resolution methods such as negotiation, mediation or arbitration to be implemented in virtual environments rather than physical ones [2], resulting in methods that save time and assets [3]. In this context, we find it important to consider the Katsch/Rifkin vision of conflict [6]. We clearly follow this approach in the sense that we aim to foster the intervention of software agents that are autonomous and act either as decision support systems or as real electronic mediators [7]. This comes near to the Second Generation ODR, proposed by Peruginelli and Chiti [7] in which technology has an autonomous role.

Based on this line of thought, in this paper we propose such architecture, aiming at a range of services useful for parties involved in legal disputes, independently of the legal domain. The architecture presented is abstract in the sense that it defines concepts and processes that are transversal. Moreover, it is also specific in the sense that it allows to implement those concepts and processes in concrete domains, in a completely transparent way. Our objective is thus to achieve an architecture based on the software agent paradigm that provides services of use to parties involved in legal disputes, independently of the legal domain. This work is being applied in three different legal fields and case studies in Portugal [11, 12, 13]: Labour Law, Consumer Law and divorce and heritage's share.

\section{Abstract Concepts and Processes}

In the field of conflict resolution, there are concepts that are independent of the legal domain addressed. Therefore, in the development of an abstract architecture, firstly one has to identify which perceptions and processes are transversal in several domains and can be modelled in an Online Dispute Resolution framework.

\subsection{Abstract Concepts}

In a first moment, a party should ask itself if it is reasonable to engage in an alternative dispute resolution process. This thought can be formalized in the concept of BATNA - Best Alternative to a Negotiated Agreement, or the possible best outcome "along a particular path if I try to get my interests satisfied in a way that does not require negotiation with the other party" [8]. This concept is transversal in the 
sense that it is valid independently of the legal domain, e.g., a party would always find it useful to know the best possible option.

Similarly, the consideration of the WATNA - Worst Alternative to a Negotiated Agreement [9] also makes sense. The WATNA defines the worst possible scenario reachable in court. However, a party might also consider the space between the BATNA and the WANTA as an additional element to consider on making or accepting a proposal, related with the concept of ZOPA - Zone of Possible Agreement proposed by Raiffa [10].

In the same line of thought, a party would also find it useful to identify the region of such space in which a result is more likely, the MLATNA - Most Likely Alternative to a Negotiated Agreement [9]. This concept denotes the most likely outcome if the parties intend to solve the conflict through litigation and may also be used by the parties to determine the best way to solve it. Extending this thought, a party may also be interested in knowing the likeliness of a group of cases distributed in the space between the BATNA and the WATNA, in an attempt to gain a better understanding of the risks and opportunities. Thus, it would be possible to consider the concept of plausible case: a possible outcome with an associated value of likeliness. When parties have access to all this knowledge, they may take better informed decisions and are in a better bargaining position.

\subsection{Abstract Processes}

Similarly to abstract concepts, there are processes whose models are transversal enough to be reused in different legal domains. Specifically, two abstract processes have been implemented in this work: case retrieval and negotiation. The objective of the first is to make a selection of past cases from a database that can be relevant according to the characteristics of the current conflict. The knowledge mentioned in the previous section is then generated from this group of cases and presented to the parties, supporting their decisions. The objective of the second process is to make available a framework of a negotiation method in which two or more parties iteratively build and reply to proposals, with the integration of the knowledge mentioned before, aiming at the reaching of a consensus on the outcome of the dispute.

\section{Building Domain-independent ODR Platforms}

In this section we detail the approach presented in this paper, concretized in two main issues: the development of an architecture abstract in nature, able to provide the necessary services, and an ontology that enables the use of these services in specific domains.

\subsection{The Architecture}

The building blocks of the architecture are a group of software agents that coordinate their functionalities in order to implement the intended services. A development strategy was followed that organizes these agents into two categories, according to their level of abstraction: high-level agents and low-level ones. In the high-level agents, we included the ones that perform tasks that do not need explicit domaindependant information. In the low-level group, we include the agents that are closer to the domain of the problem, thus making use of methodologies for problem solving and/or methods for knowledge representing and reasoning. From a general point of view, high-level agents coordinate and monitor the lifecycle of the low-level ones. Low-level agents act on the object-level data and knowledge, making the extensions of the functions that describe the universe of discourse.

To define these agents, we have taken into account the requirements on the level of the abstract processes and concepts, following and iterative cut-down process of increasing specification. The agents and their functionalities are depicted in [5].

\subsection{The Ontology}

This abstract architecture still must be specified according to each domain of use. This will be done by means of domain-specific ontologies, as depicted here. In this context, an ontology is defined as the tuple $O=$ $(V, A, C, R)$ where $V$ represents the vocabulary, $A$ stands for the actions, $C$ represents the constraints and $R$ the rules.

The vocabulary is defined by all the words that describe the concepts that belong in the respective domain. Actions define how each action should be executed, according to the specific domain, including the requirements for the action to be carried out. The rules, defined in terms of logic predicates, allow determining if certain conditions are true in a given moment. Rules are used to formalize legal norms, allowing software agents to infer the consequences of their application or violation. Rules are also used to define guidelines that allow agents to perform accordingly (e.g. if amount of pre-selected cases is higher than threshold, repeat pre-select with different algorithm). This approach also allows an efficient and relatively simple re-configuration of the system, by 
means of changes in the rules. Finally, constraints allow for add up of invariants to the perceptions and actions that make up the ontology.

The approach presented consists in defining ontologies that encode the domain-specific knowledge about the functionalities that the software agents will select, according to the domain of each problem. Its main advantage is that a single agent can be used to perform a similar task in a wide range of domains, in opposition to a traditional one in which a different agent would be used in each different domain

Let us take as an example the functionality of searching for similar cases: rather than having three agents implementing three different ways to do it according to the different types of cases in each legal domain (i.e., Commercial, Family and Labour), we have one single agent and three different ontologies. Doing so maximizes functionality reuse and allows for a single architecture to support services in different domains, without the need to perform changes in its structure. Moreover, the system can be extended without interfering with the architecture, by adding new ontologies.

\section{Results}

In this section we describe two functionalities that have been implemented with the proposed method in three different legal domains: knowledge retrieval and mediation. The Knowledge retrieval functionality implements a set of algorithms that search the casebase for cases similar to the case of the request. It returns the most similar known cases, togheter with the solution and the outcome. The mediation functionality implements a domain-independent negotiation algorithm under the blackboard paradigm, i.e., there is a shared space for message exchange, in which the parties and the system can post proposals for solving the dispute.

In Figure 1 the prototype of an interface showing the knowledge retrieved is depicted. The most important information shown are the retrieved cases, displayed in the form of colored circles in a cartesian plane. In this plane, the $\mathrm{X}$ axe represents the Utility while the $\mathrm{Y}$ axe represents the Similarity.

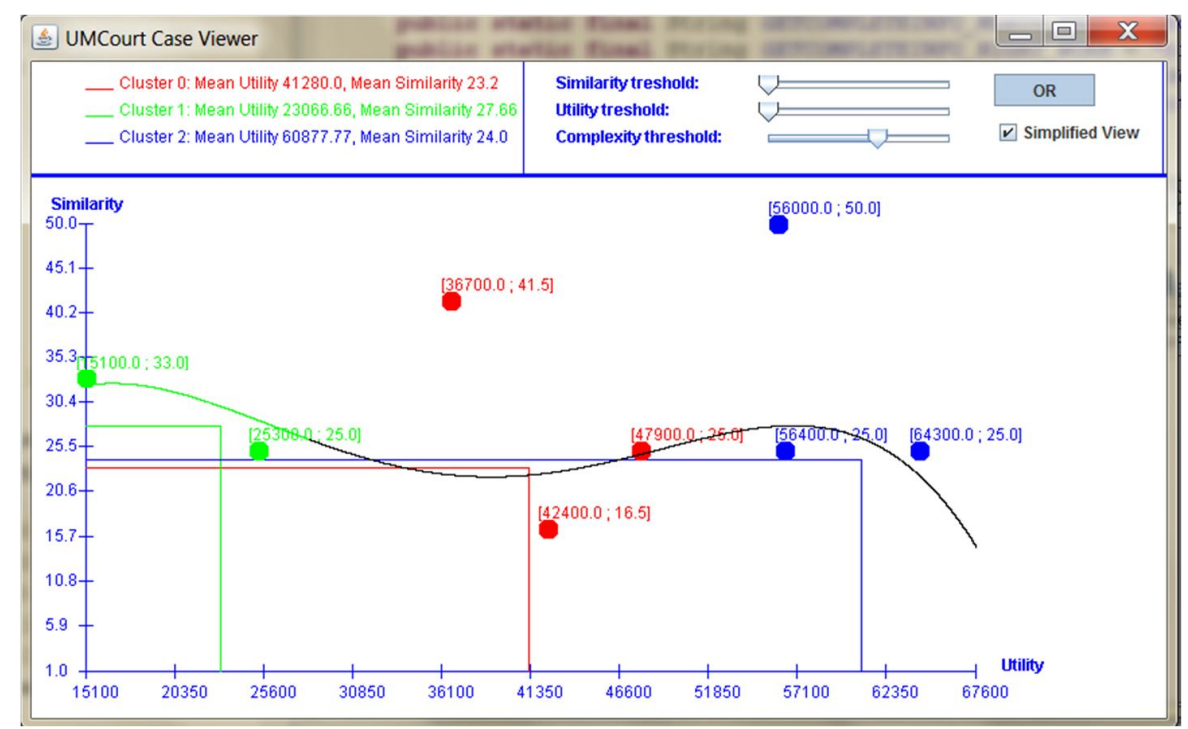

Figure 1. The prototype of the interface that shows the knowledge compiled.

Thus, the user can analyze the possible outcomes and make an evaluation of similarity (representing the likeliness to happen in court) versus the utility (denoting how desirable the outcome is). Moreover, the user can also see the BATNA, WATNA and MLATNA, represented by the region in which the curve of the linear regression is green, and some clusters with respective mean similarity and utility, that enable a general view. This is a good example of the advantages of this approach as the different ontologies define how the similarity and utility should be determined for each legal domain, whereas the display of all the information is independent of the domain.

Similarly, a mediation process can generally be seen as a sequence of steps in which the parties make proposals and, eventually, a neutral intervenes, regardless the domain. In that sense, we were able to define a mediation process based on the blackboard paradigm, in which the parties and the system can post and work on proposals for the outcome of the dispute. 
The only domain dependent information is the content of the messages, which is defined by the ontology. So, the mediation starts with the system proposing an outcome, selected from the similar cases according to criteria defined in the rules. As an example, the system can start by proposing the most likely outcome if the parties decide to go to court (the outcome of the most similar case) or start with an outcome that maximizes the probable acceptance of the parties (the outcome that maximizes the utilities). From this point on, the parties can exchange messages with new or modified proposals and, whenever they reach an impasse, the system may intervene with a different proposal, creating a new path. Figure 2 shows the prototype of the interface of the blackboard agent.

\begin{tabular}{|c|c|}
\hline UMCourt Mediation Platform I Started @ Mon Jul 12 09:02:07 BST 2010 & 0 回 $x$ \\
\hline \multirow{2}{*}{\multicolumn{2}{|c|}{$\begin{array}{c}\text { Black Board Status } \\
\text { Bands: }\end{array}$}} \\
\hline & \\
\hline Current Proposal & \begin{tabular}{|l|} 
Next Round \\
\end{tabular} \\
\hline \multirow{8}{*}{$\begin{array}{l}\text { ( } \\
\text { id: } 1297849715939 ; \\
\text { date: Wed Feb } 16 \text { 09:48:35 GMT 2011; } \\
\text { proponent: ( agent-identifier name BlackBoard@TIARAC1:1099/JADE :addresses } \\
\text { (sequence http:/192.168.68.176:7778/acc )); } \\
\text { steps: (ID, Days Received, Night Work, Suplementary Work) - (1291376839092, 34, } \\
73.0,59.0) \text { - (56000.0); } \\
\text { is answer to proposal: false; } \\
\text { is new proposal: true; }\end{array}$} & Round Statistics \\
\hline & Accept $\quad 0 \%$ \\
\hline & Ignore: $100 \%$ \\
\hline & Reply: $0 \%$ \\
\hline & Propose: $0 \%$ \\
\hline & Exit: $\quad 0 \%$ \\
\hline & $\begin{array}{l}\text { Detail: } \\
\text { Party2 }\end{array}$ \\
\hline & \\
\hline
\end{tabular}

Figure 2. The prototype of the interface of the Blackboard agent, currently holding a proposal.

\section{Conclusion}

The acceptance and use of Online Dispute Resolution tools has proven to be lower than one would expect, despite its recognized advantages. The reasons for this include a lack of multi-domain tools that can address more than one legal field. In fact, most of the currently available tools address only a single domain, drastically diminishing its application. In this paper we presented an approach that targets this problem through the use of an abstract agent-based architecture that can be specialized for specific domains by means of domain ontologies. Using ontologies to specialize a single agent in several legal domains makes the architecture simpler and also makes it easier to expand it to other legal domains, by adding new ontologies. It is our conviction that this approach will foster the development of multi-domain Online Dispute Resolution Platforms, leading to an increase in the offer of these services.

Acknowledgments. The work described in this paper was developed under the TIARAC - Telematics and Artificial Intelligence in Alternative Conflict Resolution Project (PTDC/JUR/71354/2006), a research project supported by FCT (Science \& Technology Foundation), Portugal. The work of Davide Carneiro is also supported by a doctoral grant by FCT (SFRH / BD / 64890 / 2009).

\section{References}

[1] R. Goel, E-Commerce. New, Age International, 2007

[2] E. Bellucci, A. Lodder, J. Zeleznikow, "Integrating artificial intelligence, argumentation and game theory to develop an online dispute resolution environment", ICTAI2004 - 16th IEEE International Conference on Tools with Artificial Intelligence, 2004, pp. 749-754.

[3] L. Klaming, J. Van Veenen, R. Leenes, "I want the opposite of what you want: summary of a study on the reduction of fixed-pie perceptions in online negotiations", Expanding the horizons of ODR, Proceedings of the 5th International Workshop on Online Dispute Resolution (ODR Workshop'08), Firenze, Italy, 2008, pp. 84-94.

[4] J.W. Goodman, "The pros and cons of online dispute resolution: an assessment of cyber-mediation websites", Duke Law and Technology Review, 2003.

[5] F. Andrade, P. Novais, D. Carneiro, J. Zeleznikow, J. Neves, "Using BATNAs and WATNAs in Online Dispute Resolution", New Frontiers in Artificial Intelligence, K. Nakakoji, Y. Murakami and E. McCready (Eds), Jurisin 2009, Revised Selected Papers, 2010, pp. 5-18.

[6] E. Katsch, J. Rifkin, Online Dispute Resolution resolving conflicts in cyberspace, Jossey-Bass Wiley Company, San Francisco, 2001.

[7] G. Peruginelli, G. Chiti, "Artificial Intelligence in Online Dispute Resolution", Proceedings of the Workshop on the law of electronic agents - LEA, 2002.

[8] J. Notini, Effective Alternatives Analysis in Mediation: BATNA/WATNA Analysis Demystified, (http://www.mediate.com/articles/notini1.cfm), 2005.

[9] W. Steenbergen, "Rationalizing Dispute Resolution: From best alternative to the most likely one", Proceedings 3rd ODR workshop, Brussels, 2005.

[10] H. Raiffa, The art and science of negotiation: how to resolve conflicts and get the best out of bargaining, The Belknap Press of Harvard University Press, Cambridge, 1982.

[11] D. Carneiro, P. Novais, F. Andrade, J. Zeleznikow, J. Neves, "The Legal Precedent in Online Dispute Resolution". "Legal Knowledge and Information Systems", ed. G. Governatori, Proceedings of the Jurix 2009 - the 22nd International Conference on Legal Knowledge and Information Systems, Rotterdam, The Netherlands, IOS press, 2009, pp. 47-52.

[12] N. Costa, D. Carneiro, P. Novais, F. Andrade, "An Agent-based Approach to Consumer's Law Dispute Resolution", Proceedings of the 12th International Conference on Enterprise Information Systems - ICEIS 2010, 2010.

[13] D. Carneiro, P. Novais, F. Andrade, J. Neves, "Improving Mediation Processes with Avoiding Parties", New Frontiers in Artificial Intelligence - JSAI-isAI 2010 Workshops, Revised Selected Papers, T. Onoda, D. Bekki, E. McCready (Eds) Springer - LNAI, 2011. (to appear) 